\title{
Self-similar solutions of the quasilinear relaxation of a kinetic electron beam
}

\author{
Tu Khiet and H. Abdel-Gawad \\ Laboratoire de Physique des gaz et des plasmas (*), Université Paris Sud, 91405 Orsay, France
}

(Reçu le 18 décembre 1979, accepté le 26 février 1980)

\begin{abstract}
Résumé. - On étudie le problème dynamique de la relaxation d'un faisceau cinétique d'électrons. La solution en plateau à l'état asymptotique se trouve comme un cas particulier d'une famille de solutions auto-similaires exactes de l'équation d'évolution quasi linéaire.
\end{abstract}

Abstract. - We study the dynamics of the relaxation of a kinetic electron beam. The asymptotic plateau distribution is shown to be a particular case of a family of exact self-similar solutions of the quasilinear equation.

The quasilinear theory of unstable waves is probably the best known in nonlinear plasma physics as it has received extensive investigation since the pioneering works of Vedenov, Velikhov and Sagdeev and of Drummond and Pines [1, 2]. In these papers, devoted to the problem of relaxation of an electron distribution having a beam-on-the-tail, these authors arrived at the same conclusion namely that the beam is flattened out in such a way that in the final state, a plateau is formed irrespective to the detailed form of the initial beam. This conjecture seems to have been consolidated afterward by the work of Bernstein and Engelmann [3] who gave a decisive argument in favour to the above assertion. However, computational experiments do not always confirm this theoretical prediction.

The aim of this short note is to remove, even partially, the above apparent paradox. To this end, we pay special attention to study the dynamics of the relaxation of a kinetic beam first considered by Ivanov and Rudakov [4]. We show that the diffusion coefficient which is proportional to the wave spectrum and described by a nonlinear partial differential equation, has a self-similar evolution in the form $D=v^{m+2} \Phi(z)$ where $z=v^{m} t$, the various quantities are appropriately normalized, $m$ is an arbitrary parameter and $\Phi$ obeys a nonlinear ordinary differential equation which can be analysed with some details. In particular, a choice with $m=1$ leads to an entirely soluble solution presenting the only possibility of obtaining a plateau distribution in the asymptotic time limit. We now consider an electron plasma composed

(*) Laboratoire associé au C.N.R.S of a thermal population having a density $n_{0}$ and a beam characterized by a density $n_{1}$ with $n_{1} \ll n_{0}$. The thermal part is assumed to be Maxwellian with a thermal velocity $v_{\mathrm{T}}$, the beam part is centred at a velocity $U$ and extends between the values $v_{1}=U-\Delta U$ and $v_{2}=U+\Delta U$ with $U \gg v_{\mathrm{T}}$ and $\Delta U / U \gg\left(n_{1} / n_{0}\right)^{1 / 3}$ in order to satisfy the kinetic beam condition. As is well known, waves are excited in the early stage in the domain of phase velocity $v$ such that $v_{1}<v<U$ with a growth rate of the order of $\gamma_{m}=n_{1} U^{2} / n_{0} \Delta U^{2}$.

From now on, we are only concerned with the beam part whose evolution is described by the set of equations

$$
\begin{aligned}
\frac{\partial F}{\partial t} & =\frac{\partial}{\partial v} D \frac{\partial F}{\partial v} \\
\frac{\partial D}{\partial t} & =\pi \alpha v^{2} D \frac{\partial F}{\partial v} ; \quad \alpha=n_{1} \omega / n_{0} \\
D & =\frac{\pi e^{2}}{m^{2}} \int \mathrm{d} k|E(k, t)|^{2} \delta(\omega-k v)
\end{aligned}
$$

where the notations are standard. The above equations admit an invariant

$$
\frac{\partial}{\partial v}\left(\frac{D-D_{0}}{\pi \alpha v^{2}}\right)=F-F_{0}
$$

here subscripts refer to the initial state $(t=0)$. In addition the function $D$ can be described by a closed equation resulting from a combination of (2) and (4) as

$$
\frac{\partial D}{\partial t}=\pi \alpha D v^{2} \frac{\partial F_{0}}{\partial v}+v^{2} D \frac{\partial^{2}}{\partial v^{2}}\left(\frac{D-D_{0}}{v^{2}}\right) .
$$


It is worth noticing that $D_{0}$ can be neglected in the equation except when $D$ is vanishingly small. Bearing this in mind we now solve (5) separately in the region where $F_{0}$ is nonvanishing and in the region where it is negligibly small, respectively.

1) $v_{1}<v<U$. In this domain, equation (5) shows that waves are built up, by means of the linear term, and simultaneously diffused out of this domain by means of the nonlinear term. A characteristic time, $t_{c}$, of the evolution of the waves in this domain can be estimated as that required to diffuse the beam by an amount $\Delta U$ in velocity space, this yields $t_{\mathrm{c}}=\Delta U^{2} / D$. Assuming that $D$ maintains its exponential form, i.e., $D=D_{0} \exp \left(2 \gamma_{m} t\right)$, we obtain

$$
t_{\mathrm{c}}=\gamma_{m}^{-1} \log \left(2 \gamma_{m} \Delta U^{2} / D_{0}\right) .
$$

Owing to the fact that the particles are evacuated out of this domain denoted as the production range, we speculate that a stationary state will be reached at the end of the relaxation. Whenever such a state exists, it is described by integrating (5) with $\partial D / \partial t=0$ to give

$$
\frac{\partial}{\partial v}\left(\frac{D}{\pi \alpha v^{2}}\right)=F-F_{0}
$$

where the constant of integration $F$ can be identified, by comparing (7) with (4), as the asymptotic value of $F$ in the production range. We then prove that in the hypothesis of an existence of a steady state, the distribution function tends towards a constant value which will be determined later on by requiring that the distribution be continuous in the vicinity of the frontier with the next domain we now consider.

2) $v_{\mathrm{T}}<v<v_{1}$. In this domain, the function $F_{0}$ vanishes, equation (5), in terms of dimensionless variables and function reduces to

$$
\frac{\partial \Psi}{\partial \tau}=x^{2} \Psi \frac{\partial^{2}}{\partial x^{2}}\left(\frac{\Psi}{x^{2}}\right)
$$

where

$$
x=\frac{v}{U} ; \quad \tau=\alpha t ; \quad \Psi=D / U^{2} \alpha .
$$

We can easily see that (8) admits a class of self-similar solutions depending on a parameter $m$ in the form

$$
\begin{aligned}
& \Psi=x^{m+2} \Phi(z) \\
& z=x^{m} \tau
\end{aligned}
$$

and the function $\Phi$ satisfies the equation

$$
\frac{\mathrm{d}}{\mathrm{d} z} \log \Phi=m^{2} z^{\frac{1}{m}} \frac{\mathrm{d}}{\mathrm{d} z} z^{\frac{m-1}{m}} \frac{\mathrm{d}}{\mathrm{d} z}(z \Phi)
$$

which can be integrated once to give

$$
\log \left(\frac{\Phi}{\Phi_{0}}\right)=m^{2} z^{\frac{m+1}{m}} \frac{\mathrm{d}}{\mathrm{d} z}\left(z^{\frac{m-1}{m}} \Phi\right)
$$

where the constant of integration $\Phi_{0}$ is the value of $\Phi$ at $z=0$ or at $t=0$.

We now choose $m=1$ which gives for (12) a completely soluble equation. The relevant function can be seen as a nondecreasing function of the variable $z$, it starts from the value $\Phi_{0}$ at $z=0$ and tends to a constant value, $\Phi_{\mathrm{M}}$, which is a constant of integration, when $z \rightarrow \infty$. The solution to (5) can be written explicitly as

$$
\operatorname{Li}\left(\frac{\Phi_{M}}{\Phi_{0}}\right)-\operatorname{Li}\left(\frac{\Phi}{\Phi_{0}}\right)=\frac{1}{z \Phi_{0}}
$$

where $\mathrm{Li}$ denotes the logarithm integral function. The function $\Phi$ behaves in the limits of small $z$ and large $z$, respectively as

$$
\begin{array}{ll}
\Phi=\Phi_{0}+\left(\Phi_{M}-\Phi_{0}\right) \exp \left(-1 / z \Phi_{0}\right) ; & z<1 \\
\Phi=\Phi_{M}-\left(\frac{1}{z}-\frac{1}{2 z^{2} \Phi_{M}}\right) \log \left(\frac{\Phi_{M}}{\Phi_{0}}\right) ; & z>1 .
\end{array}
$$

Turning now to the distribution function which can be seen from (4) as a function of a single variable, $z$, as $F=\frac{1}{U} \frac{\mathrm{d}}{\mathrm{d} z}(z \Phi)$. Using (14), we can see that $F$ behaves in the region where it is not vanishingly small as

$$
F=F_{\mathrm{M}}\left(1-\frac{z_{m}^{2}}{z^{2}}\right) ; \quad z>1
$$

where $z_{m}=\Phi_{\mathrm{M}}^{-1}\left(0.5 \log \left(\dot{\Phi}_{\mathrm{M}} / \Phi_{0}\right)\right)^{1 / 2}$ and $F_{\mathrm{M}}=\frac{\Phi_{\mathrm{M}}}{\pi U}$.

From the above analysis, we find that the relaxation does not run simultaneously in each point of the velocity space. For a given time, $F$ is largest in the vicinity of $v_{1}$ where $z$ is largest, while it decreases abruptly to zero at a smaller velocity which constitutes the wave front whose position, $x_{m}$, can be deduced by extrapolating the large $z$ behaviour of $F$ up to the region where it is small, giving

$$
x_{m}=\frac{z_{m}}{\tau} .
$$

As a result, the function $F$, like a heat wave, propagates from $v_{m}$ towards smaller velocities. Behind the wave front, the distribution function tends to flatten according to (15) and reaches an asymptotic value $F_{\mathrm{M}}$ in the limit $t \rightarrow \infty$. We require that $F_{\mathrm{M}}=F_{\infty}$ in order to obtain a continuous solution in the vicinity of $v_{1}$.

The sketch of variation of the function $F$ is schematically plotted in figure 1 for some representative times.

The quasilinear relaxation is terminated when the wave front approaches the domain of thermal particles. Putting $x_{m}=v_{\mathrm{T}} / U$ into (16) we obtain the saturation time as

$$
t_{\mathrm{sat}}=\gamma_{m}^{-1}\left(\frac{U^{3}}{v_{\mathrm{T}} \Delta U^{2}}\right)
$$




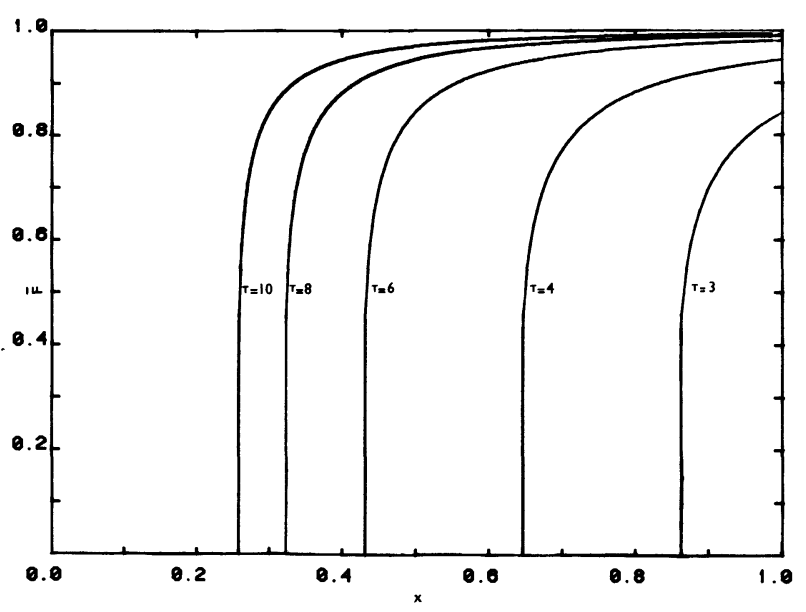

Fig. 1. - Variation of the normalized distribution function, $\bar{F}=U F$, as a function of $x=\frac{v}{U}$ in the domain of resonant velocities for several values of $\tau=\left(\frac{n_{1}}{n_{0}}\right) \omega t$.

and the final level of the distribution function deduced from the law of conservation of particles is $F_{M}=1 / U$.

Let us now compare our work with that of Ivanov and Rudakov. In our opinion our results are more rigourous for a number of points : i) In solving the diffusion equation, Ivanov and $\mathrm{Ru}-$ dakov used the approximation $v=U$ in the basic equation, consequently, their solution cannot be extended in the whole region of velocities. Their solution however, is remarkably similar to ours.

ii) Without approximating the basic equation, we have pointed the existence of many other solutions $(m \neq 1)$. Reserving the investigation of these possible solutions to a further publication, we give here only a brief comment whether or not they correspond to a physical situation. Since they behave at large $z$ as $C /\left(z^{\frac{m-1}{m}}\right)$ where $C$ is a constant of integration, it is obvious that a choice with $0<m<1$ will lead to a unphysical situation. Solutions corresponding to $m<0$ or $m>1$ are acceptable; they behave as a damped solution and do not give a plateau.

iii) We have been able to show the way to which the distribution function is flattened behind the wave front in contrast to ref. [4] where this assertion is only conjectural.

As a conclusion, a plateau solution is not a unique way towards which the distribution function evolves. Non-plateau solutions have been observed in computational experiments [5] and their existence is theoretically explained by our work.

Finally we wish to express our thanks to the referees of this article for their pertinent criticisms.

\section{References}

[1] Vedenov, A. A., Velikhov, E. P. and Sagdeev, R. Z., Nucl. Fusion Suppl. (1962) 465.

[2] Drummond, W. E. and Pines, D., Nucl. Fusion Suppl. (1962) 1049.

[3] Bernstein, I. B. and Engelmann, F., Phys. Fluids 9 (1966) 937.

[4] Ivanov, A. A. and Rudakov, L. I., Sov. JETP 24 (1967) 1027

[5] Dawson, J. M. and Shanny, R., Phys. Fluids 11 (1968) 1506. 